Dr. C. J. Lintner: Ueber d. chemische Natur d. vegetabilischen Diastase. 311

\title{
Ueber die chemische Natur der vegetabilischen Diastase.
}

\author{
Bemerkungen zu der Arbeit des Herrn cand. med. \\ Eugen Hirseh feld.
}

\begin{abstract}
Von
Dr. C. J. Lintmer, Privatdocent an der techn. Hnchschule in München.
\end{abstract}

Unter obigem Titel veröffentlichte Herr Hirs chf'eld in dieser Zeitschrift Bd. 39 p. 499 eine Arbeit, in welcher erzu dem merkwürdigen Schlusse kommt, dass die pflanzliche Diastase eine besondere moleculare Modification eines besondern Gummi sei. Vor Kurzem habe ich mun gleichfalls eine Abhandlung "Studien über Diastase" im Journal für practische Chemie N. F. Bd. 34, p. 378 erscheinen lassen, welche ungefähr denselben Gegenstand betrifft, nur dass ich es nach dem gegenwärtigen Stande unserer Kenntnisse nicht für angemessen erachtete, ein bestimmtes Urtheil über die chemische Natur der Diastase abzugeben. Bei der raschen Folge unserer Abhandlungen konnte Herr Hirsehfeld von den Resultaten meiner Arbeit, für die ich nebenbei bemerkt 1 Ctr. Grïnmalz in Angriff nahm, vermuthlich keine Kenntniss haben, sonst hätte er daraus entnehmen miissen, dass ich durch möglichst exacte quantitative Versuche bewiesen habe, dass die Diastase stickstoffhaltig ist, in ihrer Zusammensetzung jedoch von den Eiweisskörpern bei sonst grosserAehnlichkeit mit denselben wesentlich abweicht. Die nächstliegende Annahme war daher, dass die Diastase, wie das $\mathrm{H}$ ï $\mathrm{f}-$ ner bereits für die Pankreasdiastase ausgesprochen, ein $\mathrm{Oxyda-}$ tionsproduct gewisser Eiweisskörper darstelle. Ich habe 
mich nun nicht, wie Herr Hirschfeld, damit begnügt, die Wirksamkeit meiner Diastasepräparate nach dem Augenschein zu beurtheilen, wobei man, wie ich mich oft genug überzengte, groben Täuschungen ausgesetzt ist, sondern ich babe die Fermentwirkung stets quantitativ ermittelt; denn nur in der quantitativen Ermittlung des Wirkungswerthes konnte man ein sicheres Kriterium für den Erfolg der Reinigungsmethoden und für die Reinheit des Präparates gewinnen. Ja der Mangel einer quantitativen Bestimmung des Wirkungswerthes macht meiner Ansicht nach die sämmtlichen bisherigen Arbeiten über die Zusammensetzung der Diastase nahezu werthlos. Von einem sorgfältig gereinigten und mit der höchst erreichbaren Wirksamkeit ausgestatteten Präparate wurde dann durch Elementaranalyse die Zusammensetzung ermittelt. Ich will nun nicht behaupten, dass es mir durch meine Untersuchung der Gerstenmalzdiastase gelungen wäre, die wahre Zusammensetzung der Diastase endgiltig festgestellt zu haben; dazu bedarf es noch einer ganzen Reibe von Untersuchungen insbesondere von Diastasen verschiedener Herkunft, womit ich zur Zeit beschäftigt bin; das aber glaube ich als unzweifelhaft hinstellen zu dürfen, dass die wahre Zusammensetzung der Diastase von der von mir ermittelten nicht mehr bedeutend abweichen wird und dass die Diastase zwar stickstoffhaltig aber kein Eiweisskörper ist. In letzterem stimme ich also mit Hern Hirschfeld überein; irrig ist dagegen seine Annahme, dass dieselbe ein Gummi sei. Dass das nicht der Fall ist, habe ich in meiner Arbeit hinlänglich bewiesen. Ich könnte daher mit dem Hinweis auf meine Untersuchungen hier abbrechen. Ich halte es jedoch für angezeigt, Herrn Hì schfeld darauf aufmerksam zu machen, dass er bei seiner Arbeit denn doch mit zu wenig Vorsicht verfubr. Wenn es sich darum handelt, die chemische Natur eines so räthselhaften Körpers wie die Diastase a ufzuklären, begnügt man sich nicht mit der Vornahme von einer Reihe trügerischer Reactionen, sondern man bedient sich des in diesem Falle einzig zuverlässigen Hilfs mittels der Analyse und verfährt möglichst quantitativ. Hätte Herr Hirschfeld seine Diastase nach der vermeintlichen Reinigung in Substanz dargestellt und analysirt, so hätte er zweifel- 
los Stickstoff gefunden. So aber argumentirt Herr Hirschfeld ungefähr folgendermassen: die Diastase ist kein Eiweisskörper, folglich ist sie ein Gummi. Wie Herr Hirschfeld zu Aufstellung des Schlusssatzes kommt: „was wir als Diastase ansprechen ist ein Körper mit allen Reactionendes Gummi etc.", das ist mir gänzlich unerfindlich. Vergeblich habe ich zu ergriunden gesucht, was Herr Hirsehfeld eigentlich mit, ,allen Reactionen des Gummi" meint. Dass die mit Natronlauge ausgefällten Flocken von Kupferhydroxyd sich beim Kochen nicht schwärzten, kann Herr Hirschfeld doch nicht im Ernst als characteristische Gummireaction hinstellen wollen, da es bekanntlich eine ganze Reihe von organischen Substanzen giebt, welche das Schwarzwerden resp. die Wasserabspaltung aus dem Hydrat verhindern oder welche mit Kupferhydroxyd Verbindungen geben. Dass die Lösung optisch inactiv war, spricht eher gegen das Vorhandensein von Gummi; ich kenne wenigstens kein optisch inactives wahres Gummi. Die Lösung meiner Diastase erwies sich übrigens, wie ich hier bemerken will, gleichfalls als optisch inactiv. Dass der stärkeverwandelnde Körper, nicht diffundirte, kann ebensowenig als Beweis für die Gumminatur angeführt werden. Giebt es doch noch zahlreiche Körper die weder Gummi noch Eiweiss sind und nicht diffundiren. Fragen wir uns überhaupt, welches sind denn die characteristischen Eigensehaften und Reactionen der Gummiarten, so stellt sich heraus, dass dieselben sehr wenig scharf ausgeprägt sind und dass es characteristische Reationen, welche uns gestatten Gummi in Lösung ron jedem beliebigen andern Körper scharf zu unterscheiden, ub erhaupt nieht giebt. „Die Gummiarten haben die empirische Zusammensetzung $\mathrm{C}_{6} \mathrm{H}_{10} \mathrm{O}_{5}$. Man versteht darunter in den Pflanzen sehr verbreitete amorphe, durchsichtige Substanzen, die mit Wasser schon in der Kälte klebende Flïssigkeiten bilden und durch Alkohol gefällt werden. Sie sind geruch- und gesehmacklos. Einige von ihnen lösen sich ganz klar in Wasser auf, während andere nur aufquellen und sich durch Papier nicht filtriren lassen; erstere bezeichnet man als eigentliche Gummiarten, letztere als Pflanzenschleime. Durch verdünnte Säuren werden sie in reducirenden Zucker iubergeführt. Sie sind optisch activ." Das die erschöpfende Characteristik der Gummiarten. Wir sehen, wie hier bei den 
wenig scharf ausgeprägten Eigenschaften die Zusammensetzung wesentlich in's Gewicht fällt. Herr Hirschfeld bezeichnet seine Diastase allerdings als eine besondere Modification eines besondern Gummi, aber bei aller Besonderheit durfte man doch wenigstens verlangen, dass dieses bes ondere Gummi wenigstens die Zusammensetzung eines Gummi überhaupt aufweise. Diesen Nachweis aber ist uns Herr Hirschfeld schuldig geblieben. Auf eine Reihe trügerischer Reactionen hat Herr Hirschfeld seine Schliisse gebaut, was Wunder wenn sie sich nicht als stichhaltig erwiesen.

Ueber den physikalisch-optischen Bau des Auges von Cervus alces mas.

Von

Prof. Dr. Ludwig Mathiessen.

(Hierzu Tafel II.)

Durch gütige Vermittlung Sr. Excellenz des Preuss. Staatsministers Dr. Lueius sind mir im Herbste vorigen Jahres von Herrn Oberförster Reisch in bereitwilligster Weise die Lichter von dreien im Ibenhorster Forst zur Strecke gebrachter Elchbirsche zwecks ophthalmometrischer Untersuchungen zugesandt worden. Der erste schon bejahrte Elchbirsch kam am 23. October Abends durch Herrn Reisch zum Abschuss und wurden die Lichter in einer weiter unten $\mathrm{zu}$ beschreibenden Weise in dicht verschlossenen Gefässen verpackt und am 24. October pr. Post versandt. Diese 